\title{
Comparison of industrial and scientific CFD approaches for predicting cross wind stability of the NGT2 model train geometry
}

RESEARCH · SEPTEMBER 2015

DOI: $10.13140 /$ RG.2.1.2660.7844

DOWNLOADS

2

5 AUTHORS, INCLUDING:

\section{Moritz Fragner}

German Aerospace Center (DLR)

11 PUBLICATIONS 37 CITATIONS

SEE PROFILE
VIEW

1
Ralf Deiterding

University of Southampton

85 PUBLICATIONS 515 CITATIONS

SEE PROFILE 


\title{
Comparison of industrial and scientific CFD approaches for predicting cross wind stability of the NGT2 model train geometry
}

\author{
Moritz M. Fragner ${ }^{1}$, Keith A. Weinman ${ }^{1}$, Ralf Deiterding ${ }^{1}$, Uwe Fey ${ }^{1}$, \\ and Claus Wagner ${ }^{1,2}$ \\ ${ }^{1}$ German Aerospace Center, Institute of Aerodynamics and Flow \\ Technology, Bunsenstrasse 10, 37073 Goettingen, Germany \\ ${ }^{2}$ Technical University Ilmenau, Institute of Thermodynamics and Fluid \\ Mechanics, Ehrenbergstrasse 29, 98693 Ilmenau, Germany
}

February 6, 2015

\begin{abstract}
Safety assessments of cross-wind influence on high-speed train operation require a detailed investigation of the aerodynamic forces acting on a vehicle. European norm 14067-6 permits the derivation of required integral force and moment coefficients by experiments as well as by numerical simulation. Utilizing the DLR's Next Generation Train 2 model geometry, we have performed a case study comparing simulations with varying turbulence modeling assumptions. Because of its relevance for actual design, a focus lies on steady RANS computations, but more expensive unsteady RANS (URANS) and delayed detached eddy simulations (DDES) have also been carried out for comparison. Validation data for the exact same model configuration and moderate Reynolds numbers 250,000 and 450,000 is provided by side wind tunnel experiments. Particular emphasis is laid on simulating a yaw angle of $30^{\circ}$, for which a major vortex system on the leeward side of the train leads to sizeable uncertainties in predicted integral coefficients. At small to intermediate wind angles the flow remains attached and absolute errors in integral quantities decline with decreasing yaw angles. However, a consistent relative difference to the experimental results greater than $10 \%$ raises doubts about the general reliability of CFD methods, that are not capable of capturing laminar-turbulent transition, which is observed for scaled models in industry type wind tunnel experiments.
\end{abstract}


Keywords: Trains aerodynamics, cross-wind stability, drag prediction, computational fluid dynamics, experiments, vortex shedding, RANS, URANS, DDES

\section{Introduction}

The probability of wind-induced derailment increases with train speed, the vehicle area profile normal to the wind direction, and a reduction of vehicle mass. Since operating costs scale with vehicle weight, side wind stability issues can become critical. Documents regulating the rigorous verification of side wind stability of rolling stock are for instance the European norm 14067-6 or the standard RIL 807.0401-0449. Here, we concentrate on EN 14067-6 that requires an accurate assessment of aerodynamic forces on the vehicle, particularly the roll moment, for wind inflow angles from 0 to 90 degrees. Force and moment coefficients are assumed to be invariant with respect to the absolute train velocity and EN 14067-6 thereby focuses on vehicles without relative ground motion.

While the measurement of force coefficients for full-scale vehicles would be optimal, the norm - for obvious reasons - is geared towards small-scale models that can be tested inexpensively in wind tunnel experiments. As a possibly even cheaper testing procedure, EN 14067-6 also allows the evaluation of aerodynamic forces by means of computational fluid dynamics (CFD) simulations, either for full-scale or reduced model geometries, with or without ground motion considered. The guidelines for CFD in EN 14067-6 using RANS (Reynolds-averaged Navier-Stokes) equations are rather stringent and our objective in the current paper is to provide a case study in order to assess effort and reliability of CFD simulations for cross-wind stability as they would also typically be carried out in industry.

The investigated geometry is the front car of the Next Generation Train 2 (NGT2) configuration (cf. Fig. 1). The NGT2 is a DLR in-house high-speed concept train developed specifically to foster train-related research [1]. The wind tunnel model consists of the front car (for which forces are measured) and a streamlined body intended to replicate the aerodynamic influence of following cars on the lead car. Validation experiments have been conducted in the DLR Side Wind Test Facility Göttingen (SWG) as part of the Simulation Center of Aerodynamic Research (SCART). The CFD simulations have been obtained primarily with the steady, incompressible solver of the public available software OpenFOAM, which is presently very popular as a freely available open source RANS solver in industry. Cross verification is being provided by the DLR TAU code, which solves the compressible RANS equations. Previously published work [2] relied on computational meshes of comparably low resolution. In the current paper, significantly improved meshes are adopted. Further on, results from incompressible and weakly compressible URANS and DDES simulations for the largest Reynolds number and yaw angle are additionally considered.

Since the topic of rolling stock stability under side wind loading is of significant importance, several studies by others are available. Experimental studies have been 


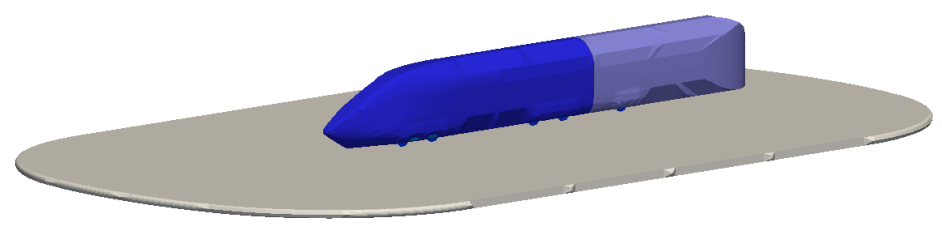

Figure 1: NGT2 front car model with streamlined end body attached to a stationary flat plate as used in the experiment.

undertaken, for instance, by Hemida \& Baker [3, 4] and by Haff et al. [5]. Numerical studies with RANS based models have been reported by Diedrichs [6] and Khier et al. [7]. Hemida \& Krajnovic [8, 9] used the large eddy simulation (LES) approach to study the influence of varying yaw angle and nose shape on flow structures around a simplified train model without inter-cap gaps and wheel bogies. Similar to the flow over a cylinder, they found that vortex shedding is induced by the shear layer instability at the periphery of the re-circulation region. In contrast to a simple cylinder, however, the nose of the train renders the flow three-dimensional at least for a distance of up to 3.5 trains heights from the tip of the nose in the direction of the length of the train. They found considerable qualitative differences between low $\left(<35^{\circ}\right)$ and larger yaw angles.

While for large yaw angles the flow is predominantly characterized by vortex shedding, for the low yaw angle set-up the flow changes from that associated with a steady slender body flow, where pairs of steady line vortices form the upper part of the wake flow, to unsteady vortex shedding after a distance of about five train heights from the tip of the nose in the direction of the train length.

This observation has been confirmed using LES simulations by Hemida \& Krajnovic [10] employing a simplified ICE2 train geometry. They study the flow at a $30^{\circ}$ yaw angle and at a Reynolds number of 200,000. In addition they identify a separate flow regime in the lower part of the wake flow, which is characterized by unsteady movement of the wake vortices, which attach and detach from the surface of the train in a regular fashion.

Dynamic flow patterns are also in agreement with the experiments by Chiu and Squire [11] that clearly exhibited unsteady vortex shedding at yaw angles $\geq 40$ degrees. A whole set of turbulence modeling techniques has been presented by Krajnovic [12] ranging from LES, hybrid RANS-LES to unsteady RANS simulations employing generic train-like vehicles. In their study hybrid RANS-LES simulations have been performed in the form of Partially-Averaged Navier Stokes (PANS) equations. 
Interesting are also the experiments by Copley [13] for yaw angle $20-35^{\circ}$ and Reynolds number of $3.7 \cdot 10^{5}$. Copley's study already identified vortices forming on the leeward side of the vehicle as a significant influence on the overall forces. When the train was modelled without wheels, a three-dimensional Karman vortex street developed, with vortices being shed from successive points on both the roof and underside of the train. However with wheels included, the vortices shed from the underside of the train were disrupted and reduced in strength, increasing the overall forces on the train.

The influence of using a stationary train model, as is used in wind tunnel experiments, as opposed to using a moving model was numerically addressed by Krajnovic et al. [14] using LES simulations. Although their simulations were performed at rather low Reynolds number $(\operatorname{Re}=22615)$ their results suggest that aerodynamic forces could exhibit significantly larger values than compared to the stationary model, which may show the limited validity of using stationary vehicles in wind tunnel experiments or CFD simulations.

Numerical simulations of trains exiting a tunnel were performed by Krajnovic et al. [15] and Krajnovic [16]. The latter study simulated an ICE2 train exiting a tunnel under the influence of a wind gust by means of detached eddy simulations. It was found that a strong vortex trailing along the upper leeward edge of the train can give rise to maximal yawing and rolling moments at the time when approximately one third and one half of the train, respectively, has exited the tunnel.

Since steady RANS simulations cannot be expected to predict highly instationary vortex shedding accurately, our CFD case study is restricted to the lower yaw angles $\theta \in\left\{0^{\circ}, 10^{\circ}, 20^{\circ}, 30^{\circ}\right\}$. For the most extreme yaw angle of $\theta=30^{\circ}$ we also conducted URANS and DDES computations in order to capture unsteady flow features which are not expected to be seen in the RANS based calculations.

The experimental and numerical setups are explained in Section 2. Note that the CFD simulations are set up to replicate the wind tunnel experiments using an end car model of 1:25 scale and the Reynolds numbers 250,000 and 450,000. The latter corresponds to approximately the maximal velocity achievable in the SWG facility. In Section 3.1, the RANS simulations are compared to the experiments in terms of force coefficients, main flow features and qualitative wall shear stress profiles. In Section 3.2, we will discuss results from URANS and DDES calculations. The conclusions are presented in Section 4.

\section{Setup}

\subsection{Experimental Setup}

The SWG facility, illustrated in Fig. 2, is a closed loop, low velocity wind tunnel with a cross section of $2.4 \mathrm{~m} \times 1.6 \mathrm{~m}$. The facility allows for wind velocities of up to $U_{\max }=65 \mathrm{~m} / \mathrm{s}$. For a typical 1:25 model, this corresponds to a maximum Reynolds 

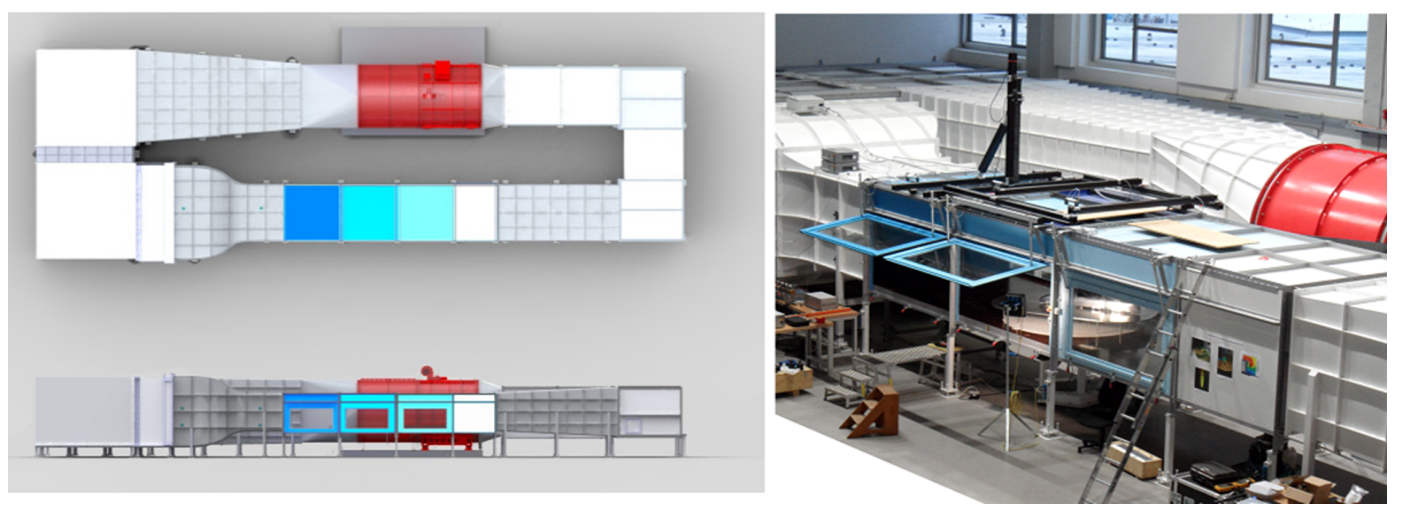

Figure 2: Wind tunnel facility for side wind experiments at DLR Göttingen.

number $\operatorname{Re}_{\max }$ of about $5 \cdot 10^{5}$.

A uniform velocity distribution in form of a block profile with relative deviations below $1 \%$ is generated inside the core region, which extends from $5 \mathrm{~cm}$ below the bottom of the vehicle to about $1 \mathrm{~m}$ above the train roof. Turbulence intensity levels are a function of the Reynolds number considered. For $\operatorname{Re}=450,000(\operatorname{Re}=150,000)$ the turbulent intensity is about $0.4 \%(0.14 \%)$ near the train surface and $0.2 \%(0.1 \%)$ in the free stream, see Fig. 3. Since the temperature inside the wind tunnel increases by up to $7^{\circ} \mathrm{C} / \mathrm{min}$ during operation, this impacts the viscosity and hence the flow velocity is monitored above the point of rotation of the model with a Prandtl probe mounted in the upper test section wall. Using this velocity, a constant Reynolds number is realized with a closed-loop rpm control. Current density and viscosity of air is determined based on the input of temperature and humidity probes located in the wind tunnel. The power of the wind tunnel compressor is then continuously adjusted to establish a constant and stable Reynolds number within a preset, maximum allowed deviation from the set-point value (typically within $0.5 \%$ ).

Data acquisition is fully automated through a coupled hard- and software system.

The train model depicted in Fig. 1 is scaled with respect to a real size train by a factor of 1:25 and has a height of $18 \mathrm{~cm}$. It is made of aluminium with a maximum surface inaccuracy of $0.05 \mathrm{~mm}$. The model is mounted onto a splitter plate with flat ground to reduce the effect of the wind-tunnel floor boundary layer. Such a flat ground configuration is compliant with the alternative ground configuration in EN 14067-6 and was used here for its simplicity. The distance between the lower edge of the wheels and the plate is about $10 \mathrm{~mm}$. Such a gap is allowed by EN 14067-6 to avoid singularities in the numerical simulations.

The train is connected to the plate via posts. The plate can be turned to allow simulation of all considered wind angles. A force gauge with a 6-component balance with integrated temperature compensation from RUAG Aviation (196-6L) is installed inside the front car to calculate aerodynamic forces from a $2 \mathrm{KHz}$ sampled time signal of a length of $5 \mathrm{~s}$. There is a small gap of $2 \mathrm{~mm}$ between the front car and the 

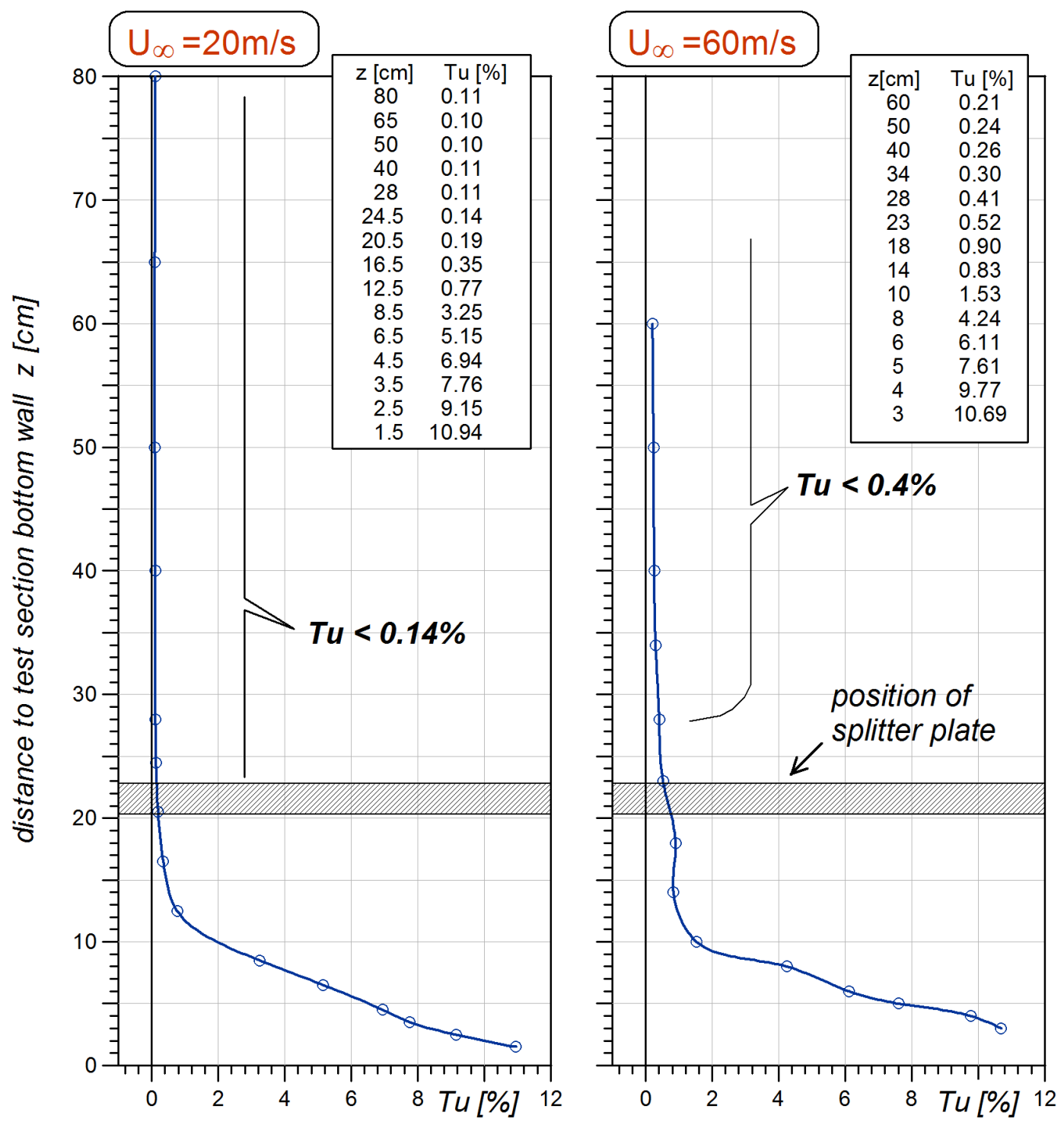

Figure 3: Turbulent intensity levels as a function of distance from the wind tunnel floor for different velocities i.e. $U_{\infty}=20 \mathrm{~m} / \mathrm{s}(\operatorname{Re}=150,000)$ and $U_{\infty}=60 \mathrm{~m} / \mathrm{s}$ $(\operatorname{Re}=450,000)$. The splitter plate is depicted and the train bottom side is at $z=$ $25 \mathrm{~cm}$.

streamline body to allow for an isolated force measurement on the front car.

Measurements of the velocity field on the leeward side of the train are conducted through particle image velocimetry (PIV). A $200 \mathrm{~mJ}$ air-cooled Nd-Yag laser with a maximum pulse frequency of $15 \mathrm{~Hz}$ is used to generate the laser light sheet. DiEthyl-Hexyl-Sebacat droplets with a mean diameter of $1 \mu \mathrm{m}$ are employed as tracer particles and images are recorded using the PCO 2000 CCD camera with a resolution of $2048 \times 2048 \mathrm{px}$. The planar PIV light sheets are oriented normal to the train length direction and 315 images are used to generate averaged PIV data. 


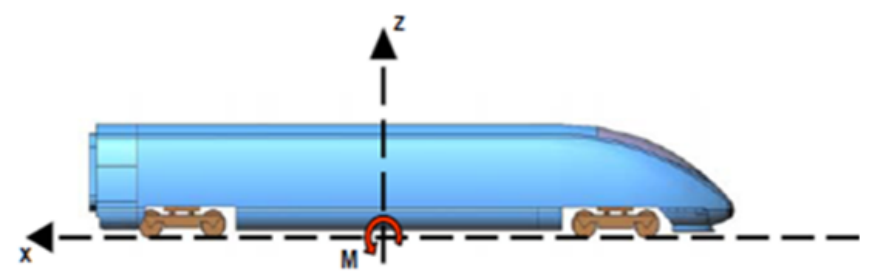

a) Side

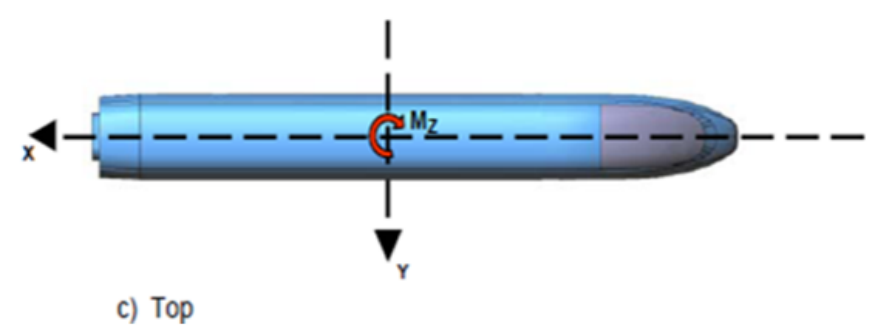

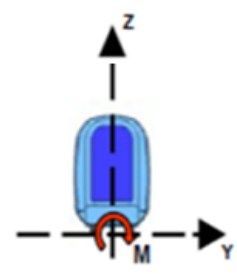

b) Back

Figure 4: Coordinate system referred to in this paper.

Additionally, wall shear stress profiles on the train and splitter plate surfaces are visualised using oil coatings. Typically it takes about $45 \mathrm{~s}$ for the oil patterns to remain steady and another $30 \mathrm{~s}$ before they become dissipated. In order to visualise laminar-turbulent transition on the train surface the method of temperature sensitive paint (TSP) is also employed $[17,18]$. The paint has a brightness sensitivity of $8 \% /{ }^{\circ} \mathrm{C}$ and has been developed in collaboration with University Hohenheim.

\subsection{Numerical Setup}

The computational investigations of the model vehicle aerodynamics are being performed with two different codes used in applied science and in industry, respectively, for comparison. As a representative for a CFD method widely used in industry, the freely available OpenFOAM system [19, 20] is employed. On the one hand it solves the incompressible Reynolds-averaged Navier-Stokes (RANS) equations together with the k- $\omega$ SST turbulence model using an implicit steady-state solver simpleFoam. On the other hand incompressible and weakly compressible URANS simulations are conducted using the implicit transient solvers pimpleFoam and rhoPimpleFoam, respectively. Additionally, DDES simulations are performed with OpenFoam using the Spalart-Allmaras DDES turbulence model in order to resolve the unsteady small-scale flow features, which are not accessible in RANS computations.

In OpenFOAM, mainly pressure corrections algorithms (cf. [21]) are available to approximate the incompressible and weakly compressible equations. The convective terms are discretised with a second order upwind scheme, diffusion terms are implemented using two non-orthogonal corrector steps for the surface fluxes, which yields second order accuracy in these terms as well. Here, the linear system arising from the pressure equation is solved with a conjugate gradient method with incomplete 


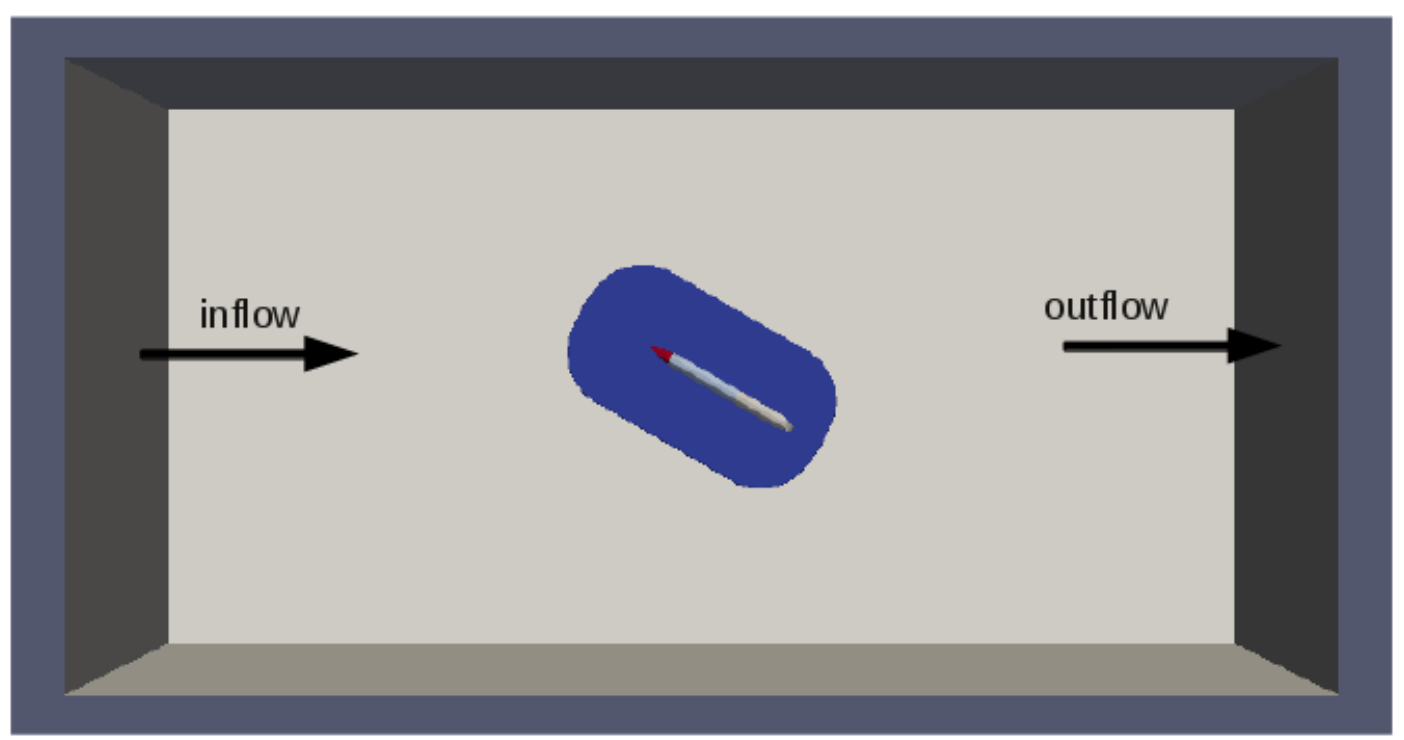

Figure 5: Illustration of inflow and outflow patches.

Cholesky preconditioning, while a Gauss-Seidel algorithm is employed for the linear systems of the other quantities.

The DLR TAU code, which is more fully described in [22], is an in-house developed fully compressible RANS solver for meeting scientific CFD standards. For this work we choose an explicit, second-order-accurate time integration scheme based on a dual-grid metric. Inviscid fluxes are computed using a central scheme of second order based on the matrix form of the Jameson-Schmodt-Turkel dissipation operator $[23,24,25]$. Viscous fluxes are handled using a second-order-accurate central discretisation operator. Finally, the Spalart-Allmaras turbulence model is used for the TAU calculations.

Fig. 4 illustrates the coordinate system used for the simulations. A simplified wind tunnel geometry is adopted (Fig.5). The computational domain boundary is sufficiently far away from the train geometry to remove the influence of far field boundary conditions and is in compliance with the simulation setup requirements formulated in norm EN 14067-6. The reference model height of $H=0.12 \mathrm{~m}$ is derived by scaling a characteristic length scale of $3 \mathrm{~m}$ by $1: 25$. The front wall is set at a distance $21 H$ from the front nose of the train. Sidewalls are placed at a distance of $\pm 21 H$ from the model. The back-wall is set at a distance of $48 \mathrm{H}$ from the back-face of the train. The top of the box was $17 \mathrm{H}$ away from the train roof, and the floor of the box was about $15 \mathrm{H}$ away from the splitter plate. A depiction of the simulation domain's dimensions is given in Fig. 5. 


\subsubsection{Mesh setup}

For OpenFOAM grid generation the snappyHexMesh mesher is employed. Fig. 6 (left panels) illustrates the mesh used in the OpenFOAM computations. Increased refinement is applied around the entire train up to a distance of $1.5 \mathrm{~cm}$ with typical grid cell length scales of $1.56 \mathrm{~mm}$.

As indicated by Fig. 6 (bottom left panel) at the solid surfaces of the train a maximum of five prism layers have been added with an expansion ratio of neighbouring layer heights of 1.2. This results in a final layer height that is comparable to half a cell size of the surrounding isotropic grid, which is $0.62 \mathrm{~mm}$, while the height of the layer located closest to the solid surfaces is as small as $0.3 \mathrm{~mm}$. This yields a dimensionless distance to the wall of $y^{+} \approx 5-30$. The upper bound of this range requires a high-Reynolds number modelling approach using wall functions. In an attempt to improve the resolution of vortices forming on the leeward side of the train increased mesh refinement is applied in a number of cylindrical regions. Typical grid cell length scales in these regions are around $1.56 \mathrm{~mm}$, see top left panel of Fig. 6.

As a high resolution reference, hybrid meshes were generated for the DLR TAU code where viscous boundaries are resolved with 20 prismatic layers, with a target dimension-less wall distance of $y^{+} \approx 1$ being achieved, see Fig. 6 (bottom right panel). The high resolution in near-wall regions of the used grids eliminates uncertainties related to wall function models. Away from the wall the typical control volume length scale before solution-based mesh refinement on the leeward side of the train is approximately $4 \mathrm{~mm}$. Note that this region is generally not as well resolved as in the OpenFoam calculations, cf. top right panel of Fig. 6. Further, TAU is a fully compressible code and thereby able to fully consider gas dynamics effects, which can be seen as a possible source of uncertainty for these computations that exhibit free stream Mach number of $M \approx 0.177$ and possibly higher values in convergent geometry details.

Already early test simulations uncovered that special attention has to be dedicated to the seemly negligible $2 \mathrm{~mm}$ gap between the front car and the streamlined end body. This gap is an unrealistic feature of the experiment, with the intention to mechanically decouple the force measurements on the front car from the end car structure. Yet, particularly for larger yaw angles flow into the gap can be considerable and a sensitivity study was conducted for the OpenFOAM setup to ensure proper computational representation. For a gap discretised with low resolution (i.e. only two isotropic cells in the gap region), we found that the overall drag coefficient was substantially lower than in the experiment. This seemed mainly driven by an artificially high pressure in the gap which caused a forward force acting on the back-face of the front car, partially neutralising the main drag force by roughly $50 \%$. Subsequently, additional cells have been added in this region and, as depicted in the middle left panel of Fig. 6, the refined mesh resolves the gap spacing with 30 cells. In this setup 10 isotropic cells plus an additional 10 boundary layer cells are used. A test calculation with the new mesh showed that the artificially high pressure region inside the gap region vanished entirely and drag coefficients much closer to the experimental result were obtained, particularly for larger yaw angles. Hence, the test calculation revealed that in a prop- 

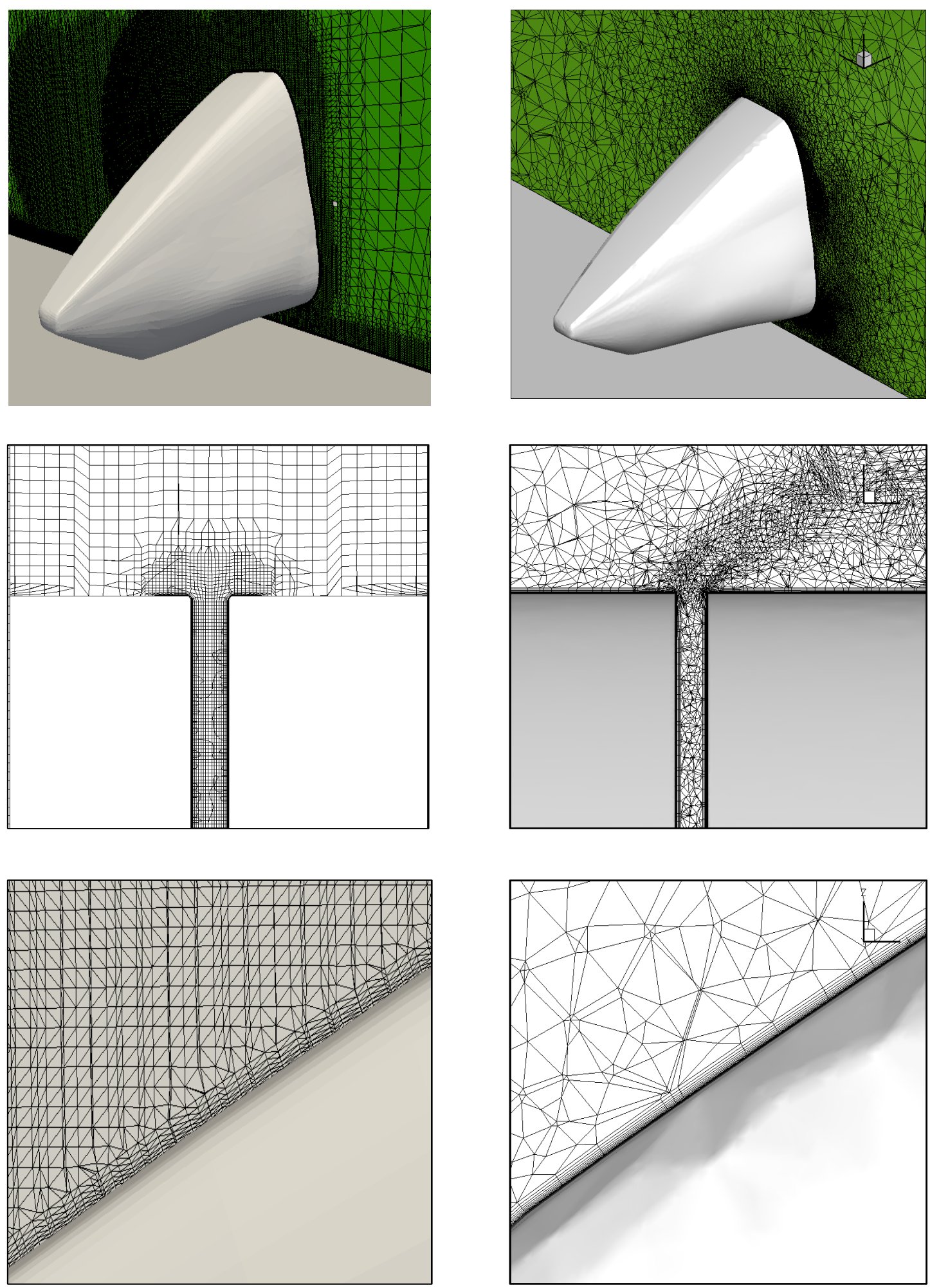

Figure 6: Grid used for the OpenFOAM (left panels) and TAU (right panels) simulations. Bottom panels: zoomed in view on the front nose boundary layer. Middle panels: zoomed in view on the gap region. 


\begin{tabular}{|l|l|l|l|}
\hline Mesh ID & N Cells (Mio) & $C_{m x}$ & $\%$ Error \\
\hline R0 & 54 & 2.8 & 9.9 \\
\hline R1 & 70 & 2.9 & 6.2 \\
\hline R2 & 82 & 3.1 & 3.1 \\
\hline R3 & 107 & 3.2 & 4.0 \\
\hline R4 & 129 & 3.2 & 4.0 \\
\hline Experiment & - & 3.078 & - \\
\hline
\end{tabular}

Table 1: Mesh refinement studies with TAU code in terms of roll moment $C_{m x}$.

erly resolved gap, the flow through the gap has a negligible effect on overall force coefficients, which motivated us to use a simplified geometry in the following OpenFOAM calculations in which the gap was removed from the model. The final mesh size for the OpenFOAM calculations consists of $\sim 64$ million cells.

As indicated before, the TAU reference computations use meshes with significantly higher local resolution. A mesh refinement study close to the requirements of EN 14067-6 has been performed, with the different meshes being tabulated in Table 1 . The maximum mesh length scale in the vicinity of the model is $0.36 \mathrm{~cm}$. In order to generate a series of mesh refinements, an adaptive mesh refinement strategy using the total pressure difference across edges as an indicator [22] is employed. This ensures that vortex cores are adequately resolved and results in an isotropic refinement factor of at least 1.5 in all three spatial dimensions in regions of high velocity gradients, as required by the standard EN 14067-6. Inside the gap region, the TAU calculations employ 40 prism layers and around 10 tetrahedra (before refinement), see middle right panel of Fig. 6. As Table 1 indicates, mesh convergence is achieved to a final relative discrepancy in roll moment $C_{m x}$ of about $4 \%$ to the experiment, which is slightly above the limit of $3 \%$ as specified in EN 14067-6.

Typical mesh generation times are up to one hour for snappyHexMesh (running in parallel mode) and up to 12 hours for the TAU meshes. It should be emphasised that both meshes themselves, although very different in resolution, are principally compliant with the norm EN 14067-6, as long as the appropriate wall treatment is being applied.

It is clear that both simulation approaches (TAU and OpenFoam) are very different, because they not only employ different implementation details of the governing equations but also utilize meshes of different resolution. As such the current study is not aimed at assessing the quality of the different codes, but rather to highlight the deficiencies when modelling cross-wind train aerodynamics using industry type simulations, latter which are represented by the low resolution OpenFOAM calculations.

\subsubsection{Boundary and Initial Conditions}

For the OpenFOAM calculations standard far field inflow/outflow boundary conditions are used, and are coupled with the hybrid wall function model provided with 


\begin{tabular}{|l|c|c|}
\hline Variable & Value & Unit \\
\hline \hline Velocity: $U_{\infty}$ & 60.25 & $\mathrm{~m} / s$ \\
\hline Pressure: $P_{\infty}$ & 99700 & $\mathrm{~Pa}$ \\
\hline Density: $\rho_{\infty}$ & 1.15 & $\mathrm{~kg} / \mathrm{m}^{3}$ \\
\hline Temperature: $T_{\infty}$ & 28.21 & ${ }^{\circ} \mathrm{C}$ \\
\hline Turbulent intensity: $k_{\infty}$ & 0.0001 & - \\
\hline Molecular viscosity: $\nu$ & $1.61 \cdot 10^{-5}$ & $\mathrm{~m} / s^{2}$ \\
\hline Ratio of $\nu_{T} / \nu$ & 10000 & - \\
\hline
\end{tabular}

Table 2: Far field conditions used in the calculations for the $\mathrm{Re}=450,000$ case.

OpenFOAM. For the compressible TAU code the low Reynolds number form of the turbulent boundary conditions are being used. Fig. 5 illustrates the location of inflow and outflow patches. Table 2 provides the far field values of the physical parameters of the computed flows and corresponds to the time averaged experimental free stream values. The far field value for velocity $U_{\infty}$ shown in the table corresponds to a Reynolds number of $R e=450 \mathrm{k}$ and is lowered for the $\mathrm{Re}=250 \mathrm{k}$ case. The far field value for the turbulent dissipation rate, $\omega_{\infty}$, is set to yield a desired ratio of turbulent to molecular viscosity, $\nu_{T} / \nu$ in the far field, as specified in Table 2 . The internal fields are initialised with these far field values as well.

The OpenFoam calculations are initialised using the velocity and pressure fields derived from potential theory. This initial condition preconditions the velocity field and generally accelerates the overall convergence of the solver. The calculations are run for a total of 14,000 iterations steps for the steady-state solver, after which the integral force coefficients are bounded to relative variations of $\sim 10^{-2}$. The global residual continuity error has converged to $\sim 10^{-8}$ in accordance with EN14067-6. This takes about 163 hours wall time on 72 cores $(11,736 \mathrm{~h} \mathrm{CPU})$ of a high-performance compute cluster consisting of compute nodes with two 6-core-Intel-Westmere processors each.

TAU computations are terminated when the variation in vehicle drag are less than one tenth of a drag count $\left(10^{-5}\right)$ and the density residual error has converged by at least 7 orders of magnitude. This convergence is achievable because of the much larger diffusion resulting from using a coarser grid in wall distant regions for the TAU calculations. On the contrary, OpenFOAM uses three times finer meshes in regions on the leeward side of the train, which seems to preserve residual unsteady effects in these calculations affecting the overall convergence of force coefficients. A typical TAU computation for the base mesh $\mathrm{R} 0$ requires 20,000 iterations and runs for about $24 \mathrm{~h}$ on 96 cores (2304h CPU) of the same parallel machine. On average, the TAU computations are a factor of about 5 faster than the OpenFOAM calculations.

Here, the URANS calculations are typically terminated after $\sim 50$ convective timescales $t_{C}=\frac{H}{U_{\infty}}$, after which time-dependent variations in force coefficients of the order of $\sim 10^{-3}$ are obtained and the global residual continuity error has converged to $\sim 10^{-12}$. The incompressible URANS computations are run with a maxi- 


\begin{tabular}{|c|c|c|c|c|c|c|c|c|c|}
\hline $\mathrm{Re}$ & $\theta$ & Method & $C_{f x}$ & $\overline{C_{f y}}$ & $\overline{C_{f z}}$ & $C_{m x}$ & $C_{m y}$ & $C_{m z}$ & $\bar{F}$ \\
\hline \multirow{12}{*}{$250 \mathrm{k}$} & \multirow{3}{*}{0} & Experiment & 0.18 & 0.0036 & -0.12 & -0.014 & 0.070 & -0.044 & 0.22 \\
\hline & & OpenFOAM & 0.21 & 0.0025 & -0.03 & 0.0014 & -0.056 & 0.013 & 0.21 \\
\hline & & TAU & 0.197 & 0.0025 & -0.078 & 0.030 & -0.09 & 0.03 & 0.21 \\
\hline & \multirow{3}{*}{10} & Experiment & 0.19 & 1.08 & 0.23 & 0.69 & -0.37 & 1.70 & 1.12 \\
\hline & & OpenFOAM & 0.22 & 1.21 & 0.22 & 0.79 & -0.33 & 2.06 & 1.25 \\
\hline & & TAU & 0.197 & 1.078 & 0.22 & 0.76 & -0.43 & 2.54 & 1.17 \\
\hline & \multirow{3}{*}{20} & Experiment & 0.09 & 2.77 & 1.20 & 1.85 & -0.97 & 3.74 & 3.03 \\
\hline & & OpenFOAM & 0.12 & 2.65 & 1.07 & 1.7 & -0.98 & 4.08 & 2.86 \\
\hline & & TAU & 0.09 & 2.3 & 1.21 & 1.9 & -1.0 & 4.2 & 2.64 \\
\hline & \multirow{3}{*}{30} & Experiment & -0.06 & 4.94 & 2.11 & 3.24 & -0.73 & 5.19 & 5.37 \\
\hline & & OpenFOAM & -0.045 & 4.22 & 2.31 & 2.69 & -1.30 & 5.56 & 4.81 \\
\hline & & TAU & -0.006 & 4.53 & 2.2 & 2.54 & -0.44 & 5.75 & 5.03 \\
\hline \multirow{12}{*}{$450 \mathrm{k}$} & \multirow{3}{*}{0} & Experiment & 0.18 & -0.0026 & -0.07 & -0.003 & -0.04 & -0.02 & 0.19 \\
\hline & & OpenFOAM & 0.21 & 0.0034 & -0.029 & 0.002 & -0.045 & 0.014 & 0.212 \\
\hline & & TAU & 0.20 & 0.0012 & -0.075 & .0 .001 & -0.06 & 0.01 & 0.213 \\
\hline & \multirow{2}{*}{10} & Experiment & 0.19 & 1.06 & 0.20 & 0.68 & -0.36 & 1.74 & 1.09 \\
\hline & & OpenFOAM & 0.21 & 1.22 & 0.27 & 0.79 & -0.37 & 2.07 & 1.27 \\
\hline & & TAU & 0.18 & 1.0 & 0.3 & 0.6 & -0.5 & 1.8 & 1.06 \\
\hline & \multirow{2}{*}{20} & Experiment & 0.11 & 2.74 & 1.09 & 1.83 & -0.91 & 3.72 & 2.95 \\
\hline & & OpenFOAM & 0.091 & 2.64 & 1.10 & 1.69 & -1.07 & 4.10 & 2.86 \\
\hline & \multirow{4}{*}{30} & TAU & 0.10 & 2.8 & 1.1 & 1.8 & -1.0 & 3.8 & 3.01 \\
\hline & & Experiment & -0.022 & 4.75 & 1.82 & 3.07 & -0.77 & 5.20 & 5.08 \\
\hline & & OpenFOAM & -0.39 & 5.32 & 2.17 & 2.99 & -0.303 & 5.85 & 5.75 \\
\hline & & TAU & -0.0199 & 4.85 & 2.1 & 2.7652 & -0.64 & 4.98 & 5.28 \\
\hline
\end{tabular}

Table 3: Table summarising force and moment coefficients.

mum CFL number of 20, corresponding to an overall runtime of 162 hours wall time on 72 cores. Due to stability issues, the weakly compressible URANS calculations are operated with a maximum CFL number of 10, and therefore overall runtimes are a factor 2.2 larger. To properly resolve high frequency structures the DDES calculations use a maximum CFL number of $\sim 1$. As a consequence, the DDES calculations are considerably more expensive and runtimes are typically a factor of 15 greater than for the incompressible URANS simulations. DDES results in here are obtained already for a total runtime of about 34 convective timescales after which the global residual continuity error has converged to $\sim 10^{-17}$.

\section{Results}

\subsection{Steady flow simulations}

In this section we discuss the main results of the steady RANS simulations and the comparison with experiments in terms of integral force coefficients, wall shear stress profiles and vorticity slices. The considered yaw angles are $\theta \in\left\{0^{\circ}, 10^{\circ}, 20^{\circ}, 30^{\circ}\right\}$ and Reynolds numbers are 250,000 and 450,000. Note that this choice satisfies the requirements of the standard EN 14067-6, which mandates cross-examination of the integral force coefficients in a minimal range of $\left[0.6 \cdot R e_{\max }, R e_{\max }\right]$.

In Table 3, integral force and moment coefficients are compared. TAU computations utilised again the mesh $\mathrm{R} 0$. The force coefficient vector $F$ is computed as

$$
F=\sqrt{C_{f x}^{2}+C_{f y}^{2}+C_{f z}^{2}} .
$$



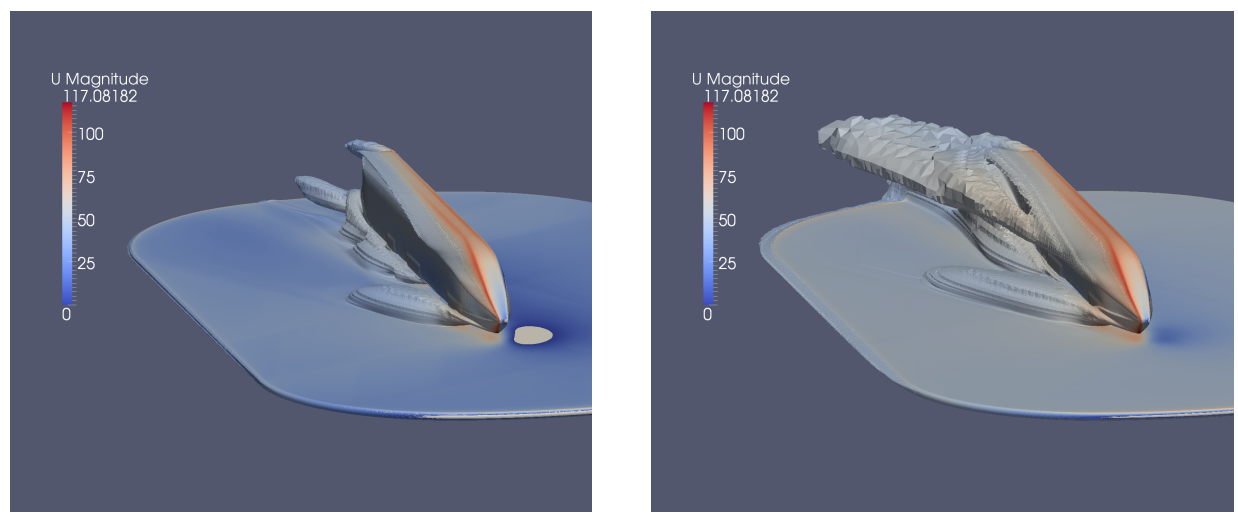

Figure 7: Iso-surfaces of pressure contour taken from an OpenFOAM calculation for the case $\theta=30^{\circ}$ and $\operatorname{Re}=450,000$. Left: $P_{t o t}=101,000$, Right: $P_{t o t}=101,500$. The color scale indicates the magnitude of flow velocity.

Force coefficients have been normalised using the dynamic pressure $q=\frac{1}{2} U_{\infty}^{2} \rho_{\infty}$ multiplied by the scaled cross sectional area $A$, where $U_{\infty}$ and $\rho_{\infty}$ are the far field velocity and density, respectively. The reference train cross sectional area is $A=$ $0.016 \mathrm{~m}^{2}$. This corresponds to a cross sectional area of $10 \mathrm{~m}^{2}$ in the $1: 1$ scale, as required by EN 14067-6. Moments are defined about the coordinate system origin (cf. Fig. 4) and are normalised by $q A H$, with $H=0.12 \mathrm{~m}$ being the characteristic length scale used in this paper.

From Table 3 it can be inferred that the drag force $\left(C_{f x}\right)$ dominates at very low yaw angles. However, as the yaw angle increases, $C_{f y}$ increases almost linearly with yaw angle and plays a critical role in determining the roll moment $C_{m x}$. The lift coefficient $C_{f z}$ also increases with yaw angle, apparently due to an increasing area of separated flow on the vehicle roof. Increasing values $C_{m x}$ and $C_{f z}$ will act to destabilise the vehicle. The Reynolds number dependency is not significant in Table 3 and this has also been confirmed by [5] and is in accordance with the requirements of EN 14067-6.

Looking at the differences between the experiment and the CFD calculations, one notices that the total force coefficient $F$ is in rough agreement with the experimental results of within $10 \%$ for most configurations. For low yaw angles $\left(\leq 10^{\circ}\right)$ the drag force naturally dominates, and we find reasonable agreement within about $15 \%$ between the numerical and experimental results for $C_{f x}$. This somehow is comparable to the experimental error bar for $C_{f x}$ of about $\pm 10 \%$ for this configuration. As the yaw angle increases, $C_{f y}$ and $C_{f z}$ gain in value and the relative agreement in $C_{f y}$ to the experiment is improving. For yaw angles greater than or equal to $\theta=30^{\circ}$, the experiments show a laminar separation bubble on the vehicle roof as clearly indicated in Fig. 11. Not unexpectedly, both RANS CFD codes cannot replicate this behaviour, with the consequence being that the discrepancies between the experiment and the computations increase visibly at $\theta=30^{\circ}$.

At this point it should be noted that the presence of such laminar flow features is 

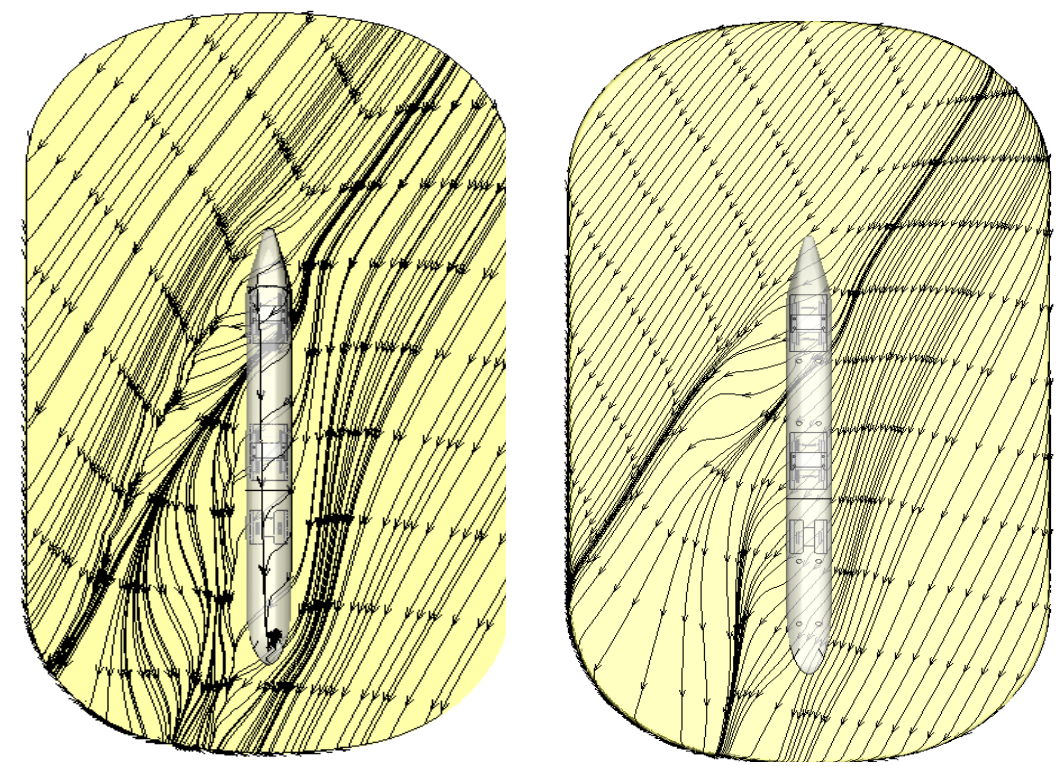

Figure 8: Wall shear stress surface streamlines on plate for the case $\theta=30^{\circ}$ and Re $=450$, 000. Left: OpenFOAM, Right: TAU.

very likely to be a consequence of using scaled wind tunnel experiments, as opposed to measuring the flow around a real size train at Reynolds numbers $\mathrm{Re}>10^{7}$. Although a suitable tripping could possibly mimic the flow under more realistic flow conditions, such tripping is typically not performed in industry. Hence the experiments in this study suggest that scaled wind tunnel measurements compliant with EN 14067-6 and typically carried out in industry, may give rise to rather unrealistic flow features. In addition such features pose extra difficulties for the numerical analysis, because numerical capturing of laminar-turbulent transition requires modelling, which may produce poor results in the presence of general complex geometries.

An interesting side observation here is that while the OpenFOAM meshes are not as well resolved as the TAU meshes in the near wall regions, the integral force and moment predictions are only marginally degraded in comparison. This indicates that the relative discrepancy between the CFD results and the experiment may not be driven by insufficient resolution in the near wall regions but could be a direct consequence of the modelling technique employed.

In the following we restrict ourselves to the most extreme configuration with $\theta=$ $30^{\circ}$ and $R e=450,000$. Fig. 7 shows iso-surfaces of two slightly different values of total pressure from an OpenFOAM calculation. For the lower value of total pressure (left panel) there is vorticity generated close to the splitter plate around the bogie sections. The first vortex, emanating from the front bogie section, is convected downstream into the wake region of the flow. The second vortex, generated at the second bogie section, is travelling with the train direction, mainly because it is bent through pressure forces induced by the low pressure region underneath the train model. Vortex footprints from these vortices can also be observed in the wall shear stress profiles of 


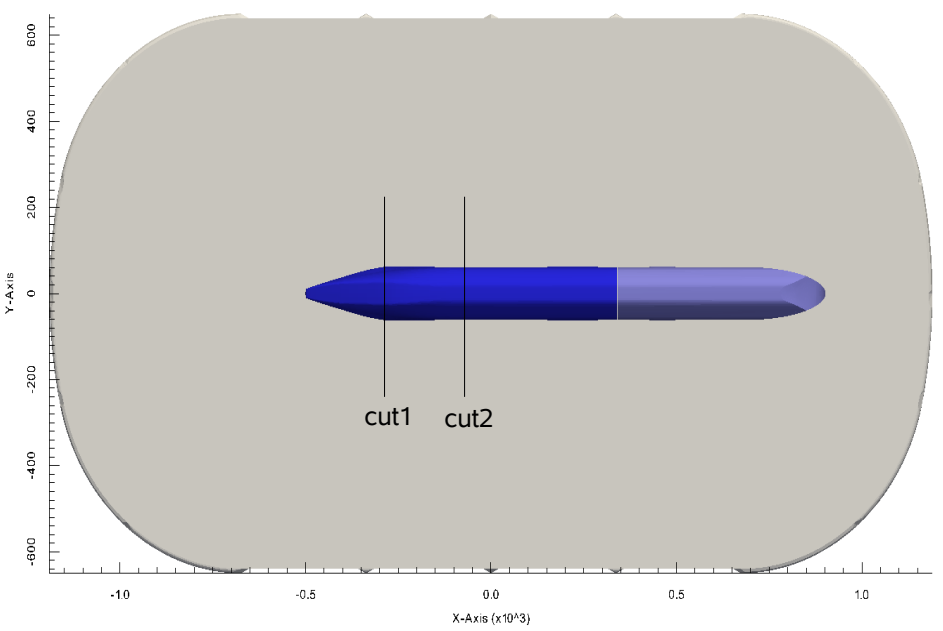

Figure 9: Locations where slide cuts were taken. The slides are perpendicular to the $\mathrm{x}$ (train length) direction. cut 1 is at $x=-290 \mathrm{~mm}$, cut2 is at $x=-80 \mathrm{~mm}$.

the splitter plate. The wall shear stress profile predictions from both codes in Fig. 8 exhibit a qualitatively similar structure, which corresponds to the footprints of the vortices seen in Fig. 7 (left panel).

From Fig. 7 (right panel) the primary vortex becomes visible. This vortex is created as part of counter rotating vortex pair at the front nose. While the lower vortex is quickly destroyed by interaction with the front bogie sections, the upper vortex stays somewhat attached to the vehicle at the roof/leeward side interface for about 3-4 characteristic length scales, cf. Hemida \& Krajnovic [8]. Since the front car has a length of $\leq 5 \mathrm{H}$, the stationarity of this vortex system justifies to some extend the use of the RANS methodology in predicting force coefficients on the front car.

In order to compare the vortex system forming on the leeward side more quantitatively among the two codes and with the experiment, we show the magnitude of $x$-vorticity (in train length direction) at two different $z-y$ planes in Fig. 10 located at the two positions as indicated by Fig. 9. The vorticity is normalised using the inverse convective time scale $t_{C}=U_{\infty} / H$. For the experiment, the vorticity data has been obtained by averaging 315 PIV images.

The primary vortex, detaching from the train roof, is captured extremely well in the OpenFOAM calculation. Its representation is somewhat degraded in the TAU simulation, which is due to utilising a mesh that is about three times less resolved in the vortex region. It is interesting to note that the OpenFOAM simulation with its better vortex resolution also seems to replicate a secondary separation near the train roof, which can also be inferred in the experimental results, that occurs as the primary vortex interacts again with train surface.

Fig. 11 presents wall shear stress surface lines on the roof of the train. The oil flow visualisation indicates the presence of a laminar separation bubble, and the TSP image also indicates laminar-turbulent transition occurring at the geometric discontinuity at 

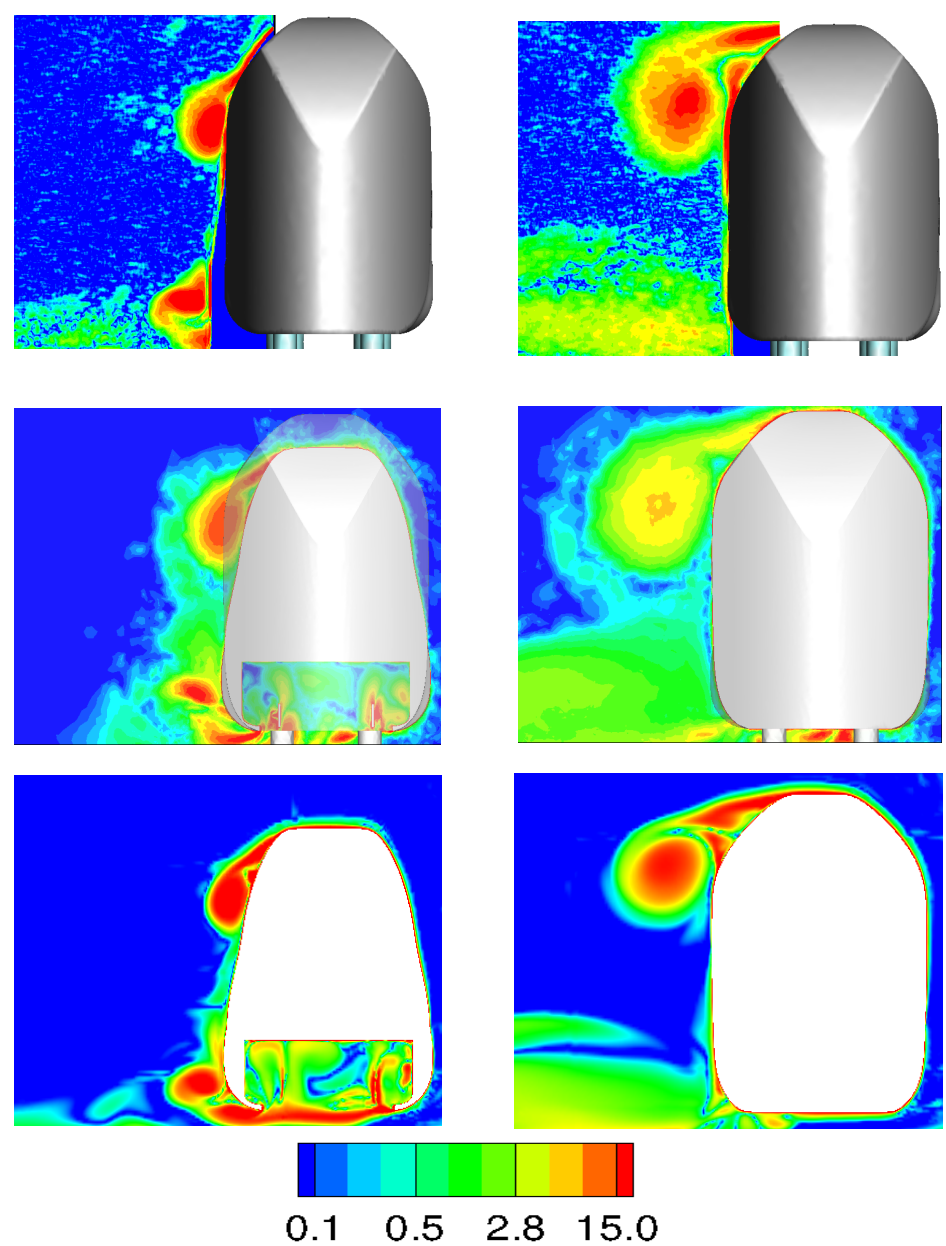

Figure 10: Magnitude of $x$-vorticity at cut 1 (left panels) and cut 2 (right panels) from PIV measurements (top), TAU code (middle) and OpenFOAM code (bottom) for the case $\theta=30^{\circ}$ and $\operatorname{Re}=450,000$.

the windward/roof interface. The RANS results (both from TAU and OpenFOAM) are unable to capture both the laminar separation bubble and the laminar-turbulent transition due to the limitations of the RANS turbulence models used. At the present time, this remains a serious weakness of all RANS based turbulence models.

A difference between the numerical predictions is also visible in Fig. 11 and is in accordance with the differences seen in Fig. 10. In the incompressible result, the separation line, which marks the point at which the primary vortex detaches from the roof into the leeward region of the train, seems to be located relatively centred on the train roof. In the experiment, this separation line can not be observed directly, however, Fig. 10 (top) suggests that it is centred on the train roof as well. In the TAU computations, on the other hand, this separation line is located further downstream and is already on the leeward side of the train, cf. Fig. 12 (middle panel). This implies that the separation of the primary vortex is delayed, which could be a compressibility effect or a consequence of employing the Spalart-Allmaras model, which is known to 

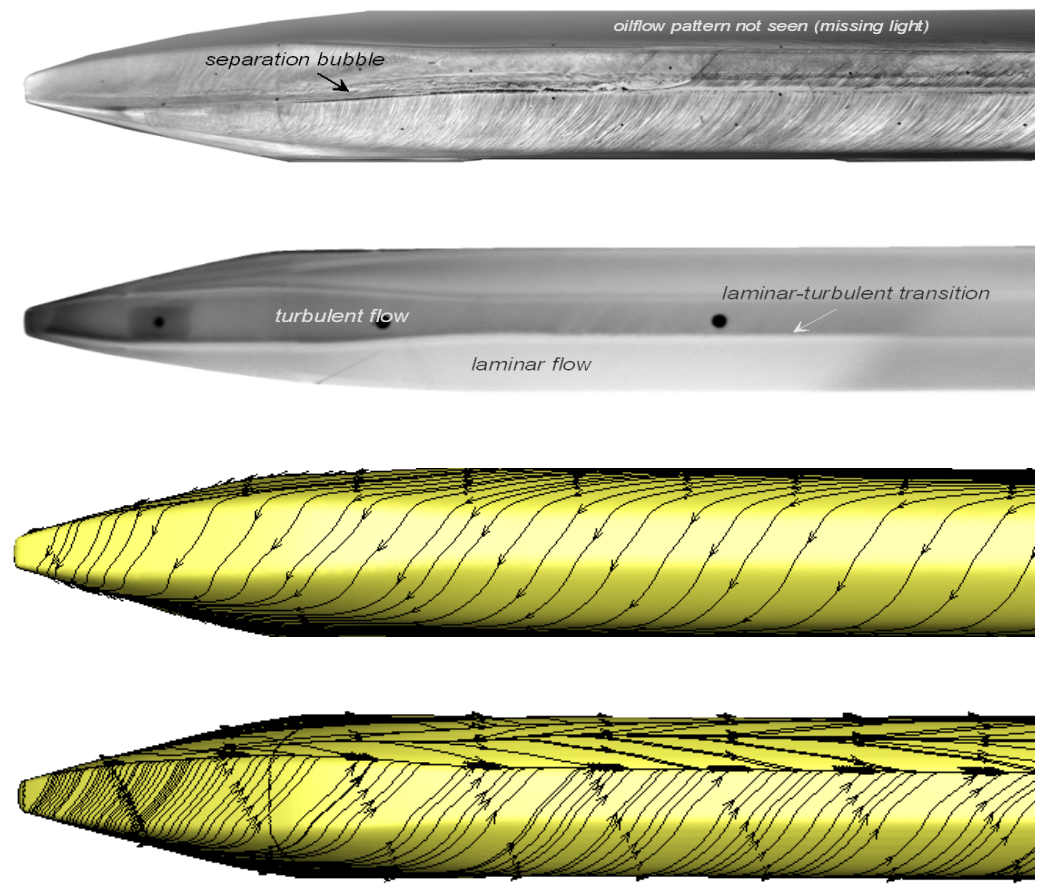

Figure 11: Wall shear stress surface streamlines on the train roof side. Top: TSP Image, second: oil flow image, third: TAU code, bottom: OpenFOAM code

exhibit artificial vortex stiffness associated with delayed separation.

Wall shear stress profiles of the leeward side are presented in Fig. 12. As discussed previously, a secondary separation line, which is a result of the primary vortex impinging again on the train roof, can be clearly identified in the experiments, cf. Fig. 12 (top panel), and the OpenFOAM simulation, cf. Fig. 10 and Fig. 12, bottom panels. This feature is not well captured in the TAU simulation, cf. Fig. 12 (middle panel), and the predicted vorticity magnitude is lower in these computations, cf. middle panel of Fig. 10, however, this is likely a result of using a computational grid for TAU that is about three times coarser in the vortex region.

Although one would expect that vortex resolution should have a noticeable influence on integral force coefficients [13], the fact that the simulation series from both codes exhibit discrepancies in integral coefficients of similar magnitude suggests that both codes suffer from modelling deficiencies that can not be explained by resolution arguments alone. It seems likely that these problems seem to be rooted in a more systematic error associated with the employed steady RANS turbulence modelling approach. 

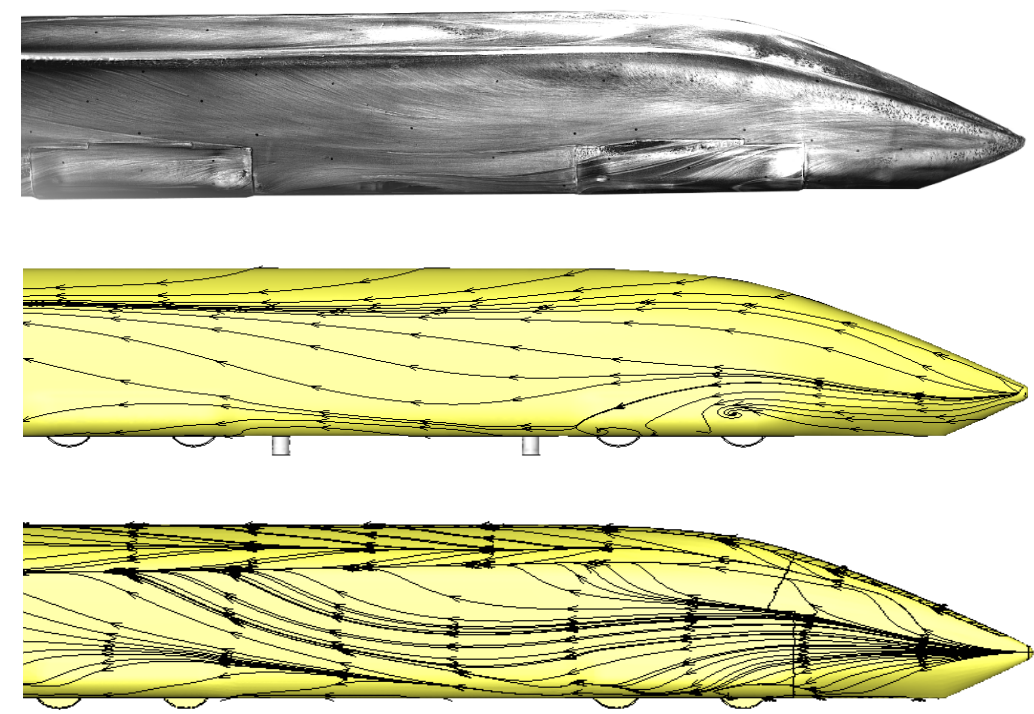

Figure 12: Wall shear stress surface streamlines of the train leeward side for the case $\theta=30^{\circ}$ and $\operatorname{Re}=450,000$. Top: Experiment, middle: TAU, bottom: OpenFOAM

\subsection{Unsteady flow simulations}

Since the previous steady RANS simulations neglect small-scale unsteady flow features, such as have been observed in the experiment and which could be responsible for driving errors in the integral force coefficient prediction, we now concentrate on time-dependent URANS and DDES simulations. Note that the time-dependent simulations in this section have been performed exclusively with OpenFOAM.

For the incompressible URANS simulations, Fig. 13 shows the vorticity component in the train length direction at three different slices together with the wall shear stress in streamline direction on the surface of the train for four different time stages. The values are normalised using the inverse of the convective timescale $t_{C}$, which is also used as the unit of time in these plots. The graphics of Fig. 13 visualise the build up of the primary vortex from the train's front nose over time.

Time slices at $t=3.2 t_{C}$ and $t=5.7 t_{C}$ show the the initial transient phase of the flow and have been added here for the purpose of reproducibility. After the initial transient phase the primary vortex structure is stabilising between $t=13.3 t_{C}$ and $t=50.9 t_{C}$ and starts to obtain a quasi-steady state, as may be seen from the fact that the flow looks similar between these slices. At $t=50.9 t_{C}$, there is again a secondary separation region visible on the surface of the train, cf. Section 3.1.

Note, however, that no unsteady small-scale flow features, such as minor separation bubbles and laminar/turbulent transition interfaces, can be observed in the URANS data. Therefore, a similar series of vorticity slices is presented for the DDES calculation in Fig. 14. In all time slices it can be seen, that small-scale turbulent eddies are 

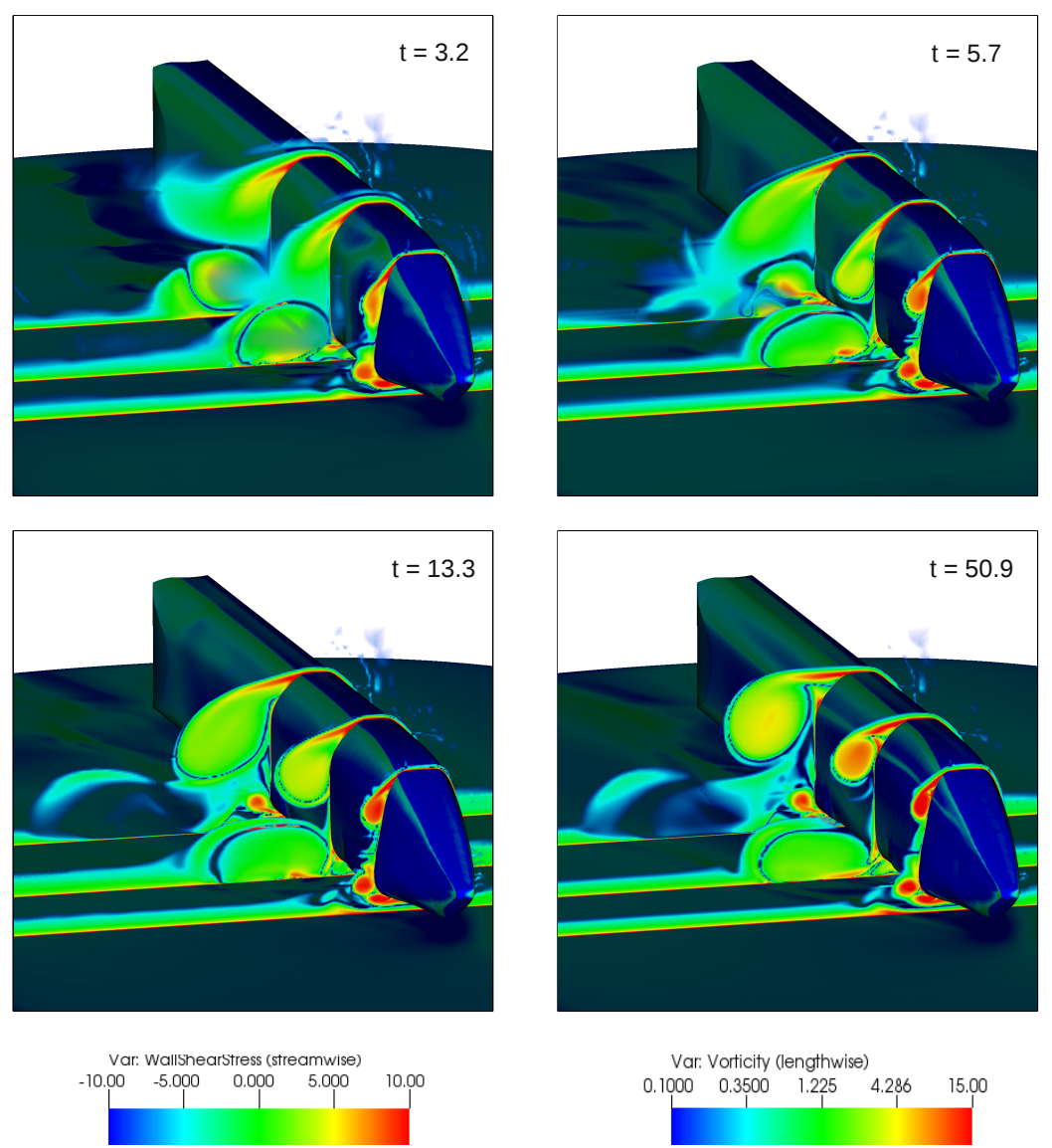

Var: Vorticity (lengthwise)
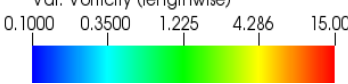

Figure 13: Instantaneous vorticity (in train length direction) at three different slices for four time stages of the incompressible URANS simulation. Also shown is the stream-wise component of wall shear stress on the train surface. Time is expressed in units of $t_{C}$.

primarily generated in the bogie sections close to the splitter plate. In contrast to the URANS computations, these eddies are preserved thanks to the relatively low turbulent diffusion associated with the DDES turbulence model. As a hybrid model, DDES applies the standard Spalart-Allmaras model only in the near-wall regions, while it behaves like a standard LES Smagorinsky model in regions further away from the wall.

Despite the fact that the flow depicted in the first slice $\left(t=9.5 t_{C}\right)$ may still be in the flow developing phase, Fig. 14 shows that the primary vortex on the leeward side of the train develops rather quickly and the entire, albeit small, primary vortex structure is already present. While the size of the vortex near the train nose is basically constant, its downstream diameter is slightly growing over time. An internal spiral structure, diffused in the URANS computations, is well-preserved. Further, numerous new small-scale eddies are present in Fig. 14 and interact with the primary vortex during its stabilisation phase at $t=25.9 t_{C}$ and $t=33.8 t_{C}$. At $t=33.8 t_{C}$, a quasi-steady 

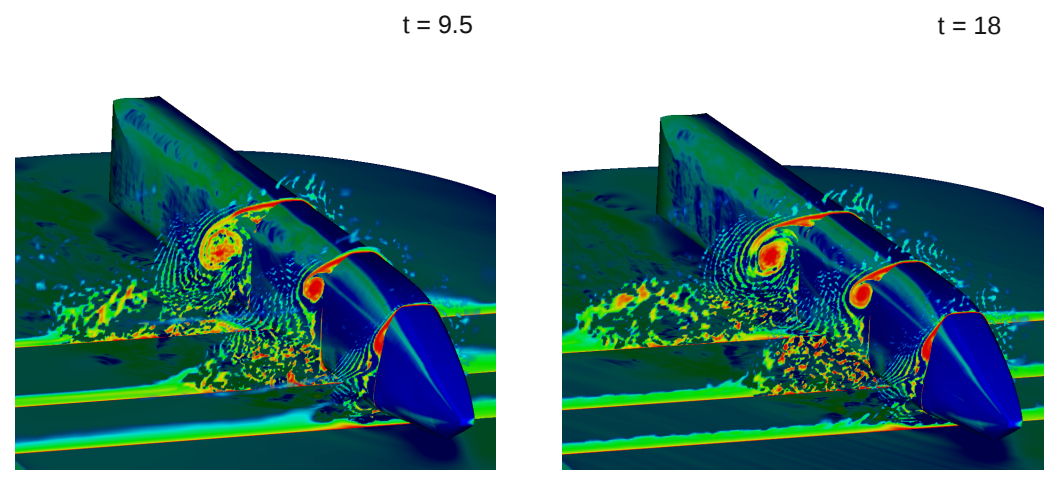

$t=25.9$

$$
t=33.8
$$
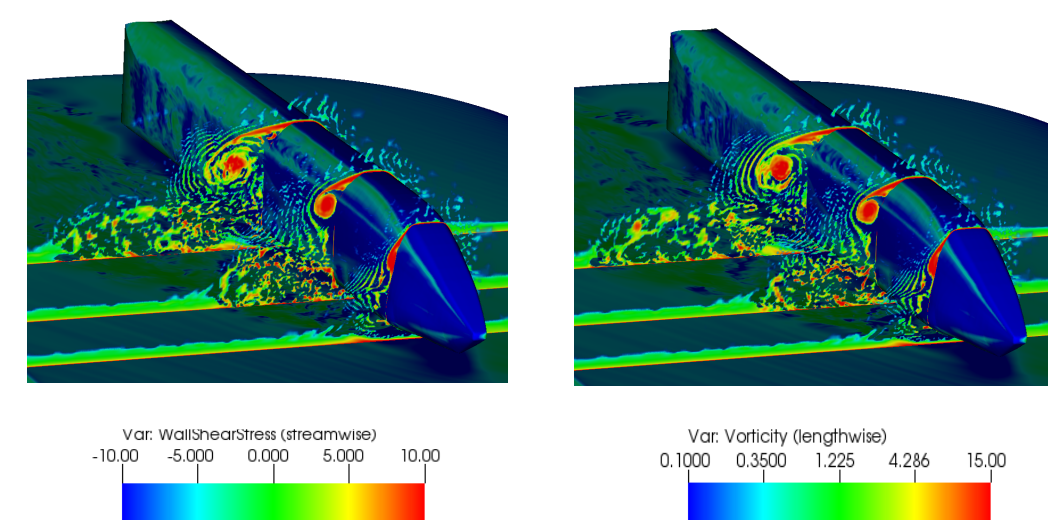

Figure 14: Instantaneous vorticity (in train length direction) at three different slices for four time stages of the incompressible DDES simulation. Also shown is the streamwise component of wall shear stress on the train surface. Time is expressed in units of $t_{C}$.

situation seems to be reached in our simulations. This observation is in agreement with [8], where it was found by LES that unsteadiness of the vortex system should not be expected below a distance of five train heights in the direction of train length, measured from the train nose.

Note that no small-scale unsteady flow features occur in the immediate vicinity of the model. This is a direct consequence of the DDES approach that still uses a standard RANS based Spalart-Allmaras model in the boundary layer. While the DDES is quite capable in resolving small-scale turbulent fluctuations on the leeward side of the train, it still can not properly model the small-scale unsteady effects on the train roof that are observed in the experiment, for instance, the small separation bubble and the exact laminar/turbulent transition location, the lack of which we accounted for the discrepancy in force coefficients.

Time averaged vorticity slices for the incompressible (top), the weakly compress- 

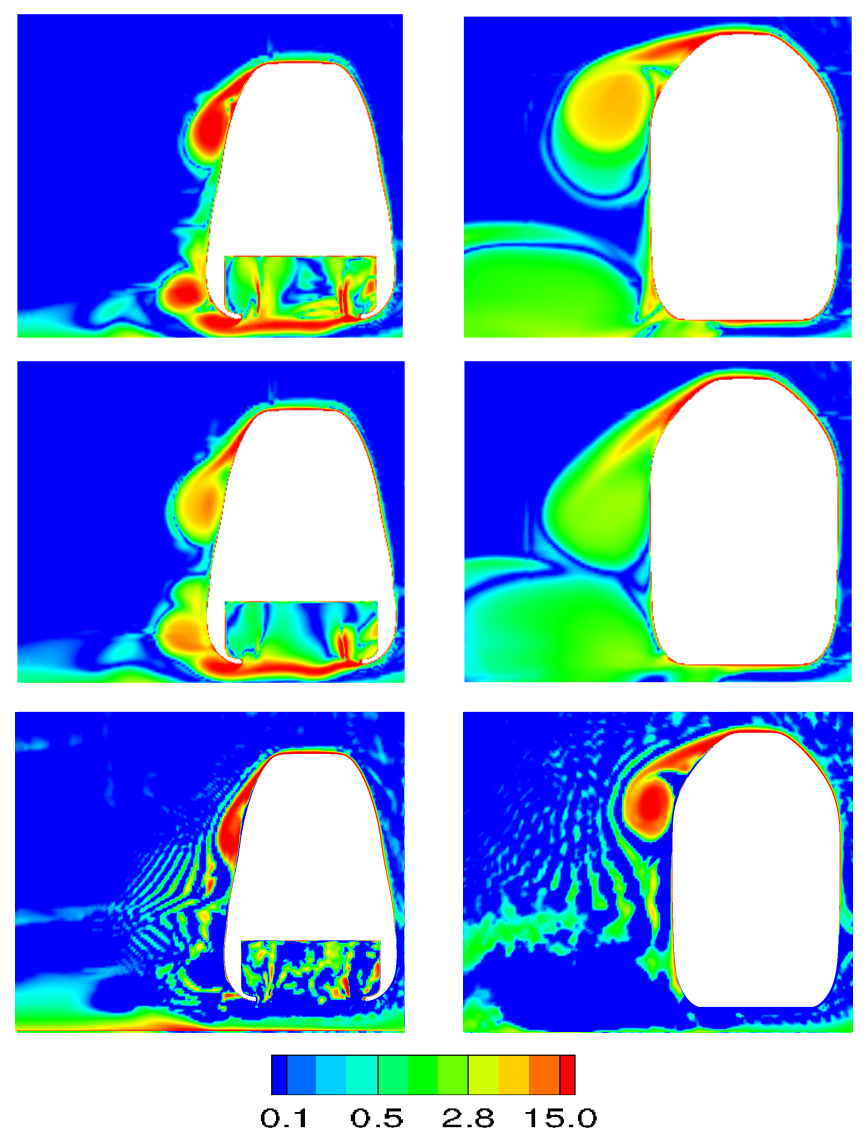

Figure 15: Time averaged magnitude of $x$-vorticity (train length direction) at cut 1 (left panels) and cut 2 (right panels) for the incompressible URANS (top), weakly compressible URANS (middle) and incompressible DDES (bottom) calculations for the case $\theta=30^{\circ}$ and $\operatorname{Re}=450,000$.

ible case (middle) and the DDES calculation (bottom) are provided in Fig. 15. These have been averaged over a time window of $\Delta t=45 t_{C}$ for the URANS simulations and $\Delta t=28 t_{C}$ for the DDES calculation. The slices correspond to the cut positions as indicated by Fig. 9 and vorticity has been normalised as before.

Comparing the time averaged incompressible URANS results (top) with the steadystate RANS computation of Fig. 10 (bottom), it can be seen that the vorticity magnitude in the unsteady case is smaller, yet, the main features can still equally be identified. Interesting differences to the incompressible case become apparent when looking at the middle panel of Fig. 15, which is showing the result of the weakly compressible simulation. Similar to the compressible TAU computations, one observes a delayed 

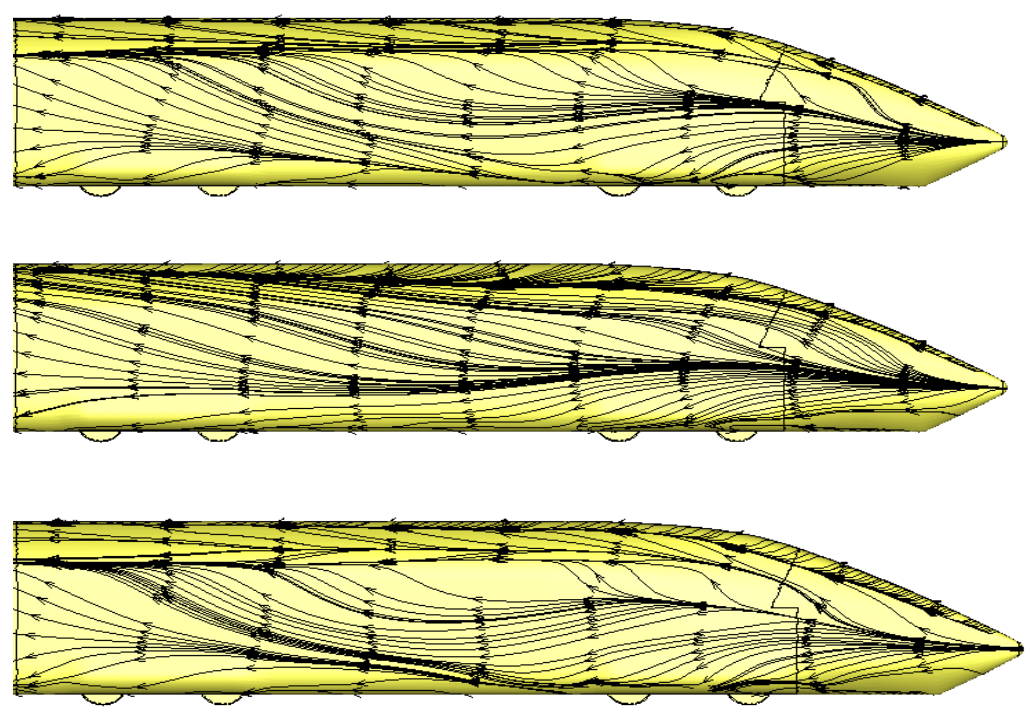

Figure 16: Wall shear stress surface streamlines on the train leeward side for the case $\theta=30^{\circ}$ and $\operatorname{Re}=450,000$ based on time averaged data. Top: incompressible URANS, middle: weakly compressible URANS, bottom: incompressible DDES

separation of the primary vortex, where the centre of rotation is lower and the vortex strength is being reduced in magnitude. It is plausible to attribute the latter to a transfer of kinetic energy in the vortex core into heat through viscous and turbulent processes, an effect that cannot be replicated by the incompressible calculations. When comparing to the experiment on the other hand, it is clear that vorticity strength seems to be better predicted in the incompressible simulation, cf. Fig. 10 (top right), which implies that compressible and weakly compressible calculations might require higher resolution in order to prevent numerical diffusion being dissipated into heat. Looking at the time averaged DDES result of Fig. 15 (bottom panel), it is clear that the vortex strength is well conserved in the back-end slice, despite the partial break up and reformation of the vortex structure observed for the different time stages, cf. Fig. 14. Although the averaging procedure for the DDES simulations may have started somewhat early and Fig. 15 may still include some signals from the initial transient flow, the primary vortex shown is very little affected by such initial conditions, because its formation occurs rather rapidly and has already established at time $t=9.5 t_{C}$.

Wall shear stress streamlines on the train surface are depicted in Fig. 16 for the leeward side and in Fig. 17 for the top of the train. Note that all these images have been generated using time averaged data. As can be seen from the visualisations, the incompressible URANS and DDES calculations both show qualitative similar streamlines, with the basic features being identical to the RANS computations discussed in the previous section. The separation line associated with the primary vortex is clearly visible from Fig. 17 and is located relatively centred on the train roof. The separation line corresponding to the secondary separation of flow moving along the train direction in the upward direction is clearly visible from Fig. 16. 

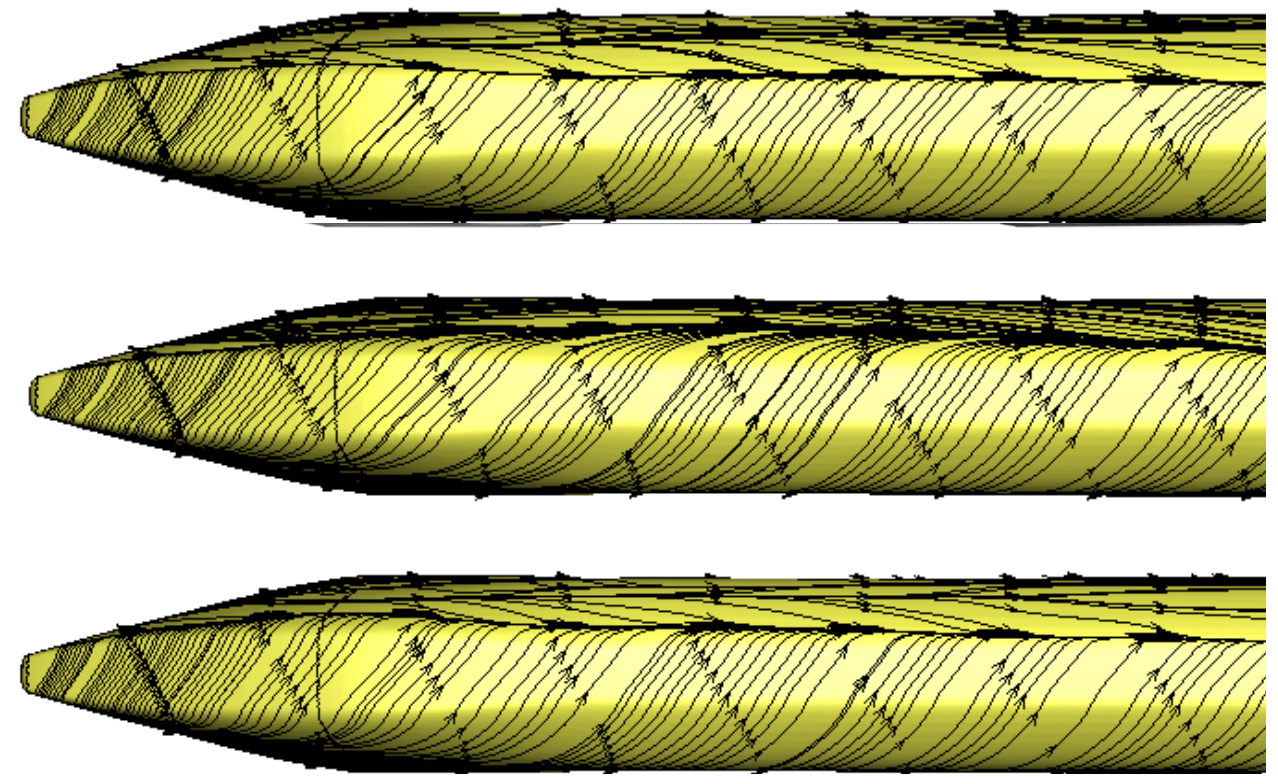

Figure 17: Wall shear stress surface streamlines on the train roof for the case $\theta=$ $30^{\circ}$ and $\operatorname{Re}=450,000$ based on time averaged data. Top: incompressible URANS, middle: weakly compressible URANS, bottom: incompressible DDES

No indication of a secondary separation line is visible for the weakly compressible calculation (middle panels) and this is a similar result to the TAU compressible RANS case. Instead only one separation line associated with separation of the primary vortex about the roof/leeward side interface can be identified. Triggered at the nose tip of the train, this separation line is located somehow lower than in the incompressible calculations, but moves in the direction of the centre of roof of the train towards the end of the front car. This is in contrast to the compressible TAU calculation, in which the height of this separation line stays somehow constant along the train length, Fig. 12. Furthermore, the streamlines on the leeward side of the train join this separation line in a much smoother fashion than is observed for the incompressible cases.

The resulting aerodynamic force coefficients are presented in Table 4. It is clear that employing time-dependent URANS simulations has not improved the prediction of aerodynamic force coefficients, with discrepancies to the experiment still being of the order of $\sim 10 \%$. For the weakly compressible calculations, the side force $C_{f y}$ and corresponding overturning moment $C_{m x}$ are generally under-predicted comparing to the experiment. Despite being capable of resolving small-scale unsteady features on the leeward side of the train, it has to be noted that the utilisation of DDES has not yet significantly improved the aerodynamic force predictions. While the moments in $y$ - and $z$-direction are matched quite accurately, the important overturning moment $C_{m x}$ exhibits a discrepancy of $\sim 20 \%$, which is worse than in all other incompressible simulations. However, in terms of force coefficients, it can be noted that the side force $C_{f y}$ is matched quite accurately. 


\begin{tabular}{|c|c|c|c|c|c|c|c|}
\hline Method & $C_{f x}$ & $C_{f y}$ & $C_{f z}$ & $C_{m x}$ & $C_{m y}$ & $C_{m z}$ & $\mathrm{~F}$ \\
\hline Experiment & -0.022 & 4.75 & 1.82 & 3.07 & -0.77 & 5.20 & 5.08 \\
OF RANS & -0.39 & 5.32 & 2.17 & 2.99 & -0.303 & 5.85 & 5.75 \\
TAU & -0.0199 & 4.85 & 2.1 & 2.7652 & -0.64 & 4.98 & 5.28 \\
OF URANS incomp. & -0.41 & 5.46 & 2.05 & 2.67 & -0.38 & 5.80 & 5.84 \\
OF URANS comp. & - & 4.01 & 2.84 & 1.80 & - & 5.30 & - \\
OF DDES & -0.4 & 4.99 & 2.78 & 2.44 & -0.72 & 5.18 & 5.72 \\
\hline
\end{tabular}

Table 4: Table summarising force and moment coefficients for the URANS/RANS and DDES calculations for the case $\theta=30^{\circ}$ and $\operatorname{Re}=450,000$.

\section{Conclusions}

A comprehensive comparison between CFD simulations and experimental measurements addressing the side wind stability of a model train front car has been presented. The investigations are oriented along the requirements of European norm 14067-6, which regulates the assessment of integral force and moment coefficients of rolling stock by means of model experiments and CFD simulations. Intentionally, only lower yaw angles with $\theta \leq 30^{\circ}$ are considered.

In terms of force and moment coefficients, the numerical results agree within a factor of $10-15 \%$ with the experimental measurements. This is significantly above the Reynolds number dependence of the experimental data that varies for instance by less than $2 \%$ in the critical roll moment $C_{m x}$ for $\theta \leq 20^{\circ}$ and by $\sim 5 \%$ for $\theta=30$, as verified by considering $\operatorname{Re}=250 \mathrm{k}$ as well as $\mathrm{Re}=450 \mathrm{k}$. This discrepancy is visible in a rather constant relative difference between the numerical and experimental results, which is attributed to the fact that laminar separation and turbulent transition effects occur in the experiment which cannot be captured correctly by any of the tested turbulence modelling techniques.

A series of industry-typical incompressible RANS simulations with the popular open source software OpenFOAM has been compared to computations with DLR's inhouse fully compressible RANS solver TAU, which seems justified as the free stream Mach number of the $\mathrm{Re}=450 \mathrm{k}$ case is $M \approx 0.177$. While the OpenFOAM simulations utilise approximately three times finer resolution than TAU to resolve leeward side vortices, the near wall regions are only resolved down to the logarithmic boundary layer, which makes usage of turbulent wall functions necessary. On the other hand, the considerably more expensive TAU computations achieve a consistent dimension-less wall distance of $y^{+} \approx 1$ and thereby remove the need for turbulent wall functions. Despite varying thereby all these crucial simulation components, it has to be observed that force and moment coefficients still exhibit major discrepancies to the experimental measurements.

A detailed analysis of vortex formation in CFD simulations and experiment has been given for the largest considered yaw angle $\theta=30$. All simulations clearly reproduce the most dominating vortices correctly and in rather good agreement with the experiments. Vortices triggered in the wheel and bogie sections can be identified via surface streamlines on the splitter plate as well as from total pressure contours. 
The primary vortex, which is initiated at the train nose and stays attached to the train surface for some distance before it starts to detach as well, can be visualised via PIV images and vorticity slices. This vortex is found to be located further away from the train surface as the yaw angles is increased, a result also found experimentally [5].

It is noted that vorticity strength preservation is much better in the incompressible simulations, while the compressible and weakly compressible simulations generally exhibit more dissipative results even on identical meshes. In particular, the vortex strength towards the end of the front car is better represented and only the incompressible simulations replicate a secondary separation line that is also observed in the experiment.

In order to assess the influence of unsteady and small-scale turbulent features on the prediction of integral coefficients, URANS and DDES computations have been carried out for the most extreme configuration with $\theta=30$ and $R e=450,000$. From the URANS simulations it is verified that the vortex system on the leeward side of the train attains a steady-state after about 50 convective timescales, which was already argued by previous authors [8] and gives some justification for using RANS based methods in the first place. Once a steady state is achieved, there is virtually no major difference in terms of force coefficients between the incompressible URANS and RANS computations. The incompressible DDES calculations, on the other hand, show interesting time-dependent features of the leeward side vortex system. However, even despite its generic ability to capture small-scale turbulent unsteady features, DDES is not able to capture the unsteady effects on the train roof prior to the separation of the primary vortex, such as the laminar separation bubble and the laminar/turbulent transition as seen in the experiment. This is most likely due to the fact that the DDES model is tuned to model the entire boundary layer with a RANS based Spalart-Allmaras model and thereby is missing the flow dynamics within the boundary layer.

In general, we attribute the lack of any of the investigated turbulence models in accurately representing flow separation and laminar-to-turbulent transition as the greatest obstacle for predicting integral force and moment coefficients within the threshold of $3 \%$ as required by EN 14067-6. While this result might seem rather disappointing at first glance, it has to be emphasised that it has been obtained for a train model of 1:25 scale and accordingly small Reynolds numbers. Particularly the laminar-toturbulent transition feature observed in the experiment would not occur for the flow around a real size train, where Reynolds numbers are a factor 20-40 larger, and the flow is fully turbulent before even reaching the train. To eliminate this unrealistic feature of the experiment, trip wires could be employed to force the flow to become fully turbulent, and thus avoiding the occurrence of laminar/turbulent transition. This could eliminate many of the difficulties associated with the numerical modelling when scaled models are simulated. 


\section{References}

[1] http://www.dlr.de/dlr/desktopdefault.aspx/ tabid-10467/740_read-916/gallery/2043

[2] M.M. Fragner, K.A. Weinman, R. Deiterding, U. Fey, C. Wagner, "Numerical and Experimental Studies of Train Geometries Subject to Cross Winds", in J. Pombo, (Editor), "Proceedings of the Second International Conference on Railway Technology: Research, Development and Maintenance", Civil-Comp Press, Stirlingshire, UK, Paper 24, 2014. doi:10.4203/ccp.104.24

[3] C.Baker, 'Some complex applications of the wind loading chain', Journal of Wind Engineering and Industrial Aerodynamics, 91, 17911811, 2003.

[4] C.Baker, J.Jones, F.Lopez-Calleja, J.Munday, 'Measurements of the cross wind forces on trains.' Journal of Wind Engineering and Industrial Aero- dynamics 92, 547563, 2004.

[5] J. Haff, J. Richard, U. Fey, T. Kowalski, S. Loose, C. Wagner, 'Wind Tunnel Experiments with a High-Speed Train Model subject to Cross Wind Conditions', in J. Pombo, (Editor): Proceedings of the First International Conference on Railway Technology: Research, Development and Maintenance, Civil-Comp Press, Stirlingshire, Scotland, 2012.

[6] B.Diedrichs, 'On computational fluid dynamics modelling of cross wind effects for high-speed rolling stock', IMechE 217(F),203226, 2003.

[7] W.Khier, M.Breuer, F.Durst, 'Flow structure around trains under side wind conditions: a numerical study' Computers and Fluids 29, 179195, 2000.

[8] H.Hemida, H. Krajnovic: 'LES study of the influence of the nose shape and yaw angles on flows structures around trains', Journal of Wind Engineering and Industrial Aerodynamics. 98, 34-46, 2010.

[9] H.Hemida, S. Krajnovic: 'LES study of the influence of train nose shape on the flow structures under cross-wind conditions', Journal of Fluid Engineering, Vol. 130, issue 9, 091101, 2008

[10] H.Hemida, S. Krajnovic: 'Exploring Flow Structures Around a Simplified ICE2 Train Subjected to a 30 Degree Side Wind Using large-Eddy Simulation', Journal of Engineering Applications of Computational Fluid Mechanics. Vol. 3, No. 1, pp. 28-41, 2009

[11] T.W.Chiu, L.C. Squire, 'An experimental study of the flow over a train in a cross wind at large yaw angles up to $90^{\circ}$, Journal of Wind Engineering and Industrial Aerodynamics 45, 4774, 1992.

[12] S. Krajnovic, 'On Unsteady Simulations in Train Aerodynamics', Railways 2014 The Second International Conference on Railway Technology: Research, Development and Maintenance Ajaccio, Corsica, France 8-11 April 2014.

[13] J.M.Copley, 'The three-dimensional flow around railway trains', Journal of Wind Engineering and Industrial Aerodynamics 26,2152, 1987.

[14] S.Krajnovic, P. Ringqvist ; K. Nakade et al.: 'Large eddy simulation of the flow around a simplified train moving through a crosswind flow.', Journal of Wind Engineering and Industrial Aerodynamics. 110 s. 86-99, 2012 
[15] S.Krajnovic, E.Bjerklund, B. Basara : 'Simulation of the flow around high-speed trains meeting each other and at the exit of a tunnel', 21st International Symposium on Dynamics of Vehicles on Roads and Tracks, Stockholm, Sweden, 17-21 August, 2009.

[16] S.Krajnovic: Computer Simulation of a Train Exiting a Tunnel Through a Varying Crosswind. International Journal of Railway . 1 (3 (2008.9)) s. 99-105.

[17] U. Fey et al.,'Investigation of Reynolds number effects in high-speed train wind tunnel testing using temperature-sensitive paint', 2nd symposium on RailAerodynamics 2013, Berlin; 15-17, May 2013.

[18] Tropea et al. [Ed.], 'Transition detection by Temperature-Sensitive Paint', Springer Handbook of Experimental Fluid Mechanics, Chap 7.4, Springer, Heidelberg, 2007.

[19] H.G. Weller, G. Tabor, H. Jasak, and C. Fureby. 'A tensorial approach to computational continuum mechanics using object orientated techniques', Computers in Physics, 12(6):620 - 631, 1998

[20] H. Jasak. Error analysis and estimation in the Finite Volume method with applications to fluid flows. PhD thesis, Imperial College London, 1996.

[21] J.H. Ferziger, M. Peric, 'Computational Methods for Fluid dynamics', Springer, 1997.

[22] D. Schwamborn, T. Gerhold, R. Heinrich. 'The DLR TAU-code: recent Applications in Research and Industry", European Conference on Computational Fluid Dynamics, Egmond aan Zee, ECOMASS CFD, 2006.

[23] A. Jameson, 'Artificial diffusion, upwind biasing, limiters and their effect on accuracy and multigrid convergence in transonic and hypersonic flows', 11th AIAA CFD Conference, Orlando, 1993.

[24] R. C. Swanson, R. Radespiel and E. Turkel, 'Comparison of several dissipation algorithms for central difference schemes', ICASE Report No. 97-40, 1997.

[25] R. C. Swanson, E. Turkel, 'On central difference and upwind schemes', JCP, 101:292-306, 1992. 